\title{
Uso de fármacos y productos naturales antiparasitarios por madres de familia de niños y niñas en etapa escolar, Chachapoyas - 2019
}

\section{Use of drugs and natural antiparasitic products by mothers of children in school, Chachapoyas- 2019}

Carla M Ordinola R ${ }^{1}$, Yshoner A Silva D², Oscar J Oc ${ }^{3}$, Shírley J Tuesta M ${ }^{4}$ y Oscar Pizarro $\mathrm{S}^{5}$

\begin{abstract}
RESUMEN
La presente investigación tuvo como objetivo comparar el uso tanto de los fármacos y los productos naturales antiparasitarios. La muestra fue de 115 madres de niños y niñas en etapa escolar. Se obtuvo que el $30.4 \%$ de madres utiliza fármacos antiparasitarios, el $63.5 \%$ utiliza fármacos y productos naturales antiparasitarios y el $6.1 \%$ utiliza productos naturales antiparasitarios. Los productos naturales utilizados son: paico y "leche de ojé" en un $27 \%$, paico y semilla de papaya en $17.4 \%$, bebida tibia de hierba buena con leche y uso del paico en un $14.8 \%$, semilla de calabaza y bebida tibia de hierba buena en $10.4 \%$. La forma de adquisición de conocimiento de las madres sobre las propiedades antiparasitarias de los productos naturales, son: el $58.3 \%$ posee conocimiento transmitido de familiares y amigos. La preferencia entre el uso de fármacos y el uso de productos naturales antiparasitarios, el 20.9\% de madres prefieren el uso de fármaco por la facilidad de administración e indicación médica. Así mismo 58.3\% prefieren los productos naturales por uso y recomendación de familiares. En conclusión, la mayoría de madres utiliza los productos naturales y los fármacos para la desparasitación de sus hijos.
\end{abstract}

Palabras clave: Fármacos, antiparasitarios, plantas medicinales.

\begin{abstract}
The present investigation of quantitative approach, comparative level, cross-sectional. It aimed to compare the use of drugs and the use of natural antiparasitic products. The sample was 115 mothers of boys and girls in school. In the results: $30.4 \%$ of mothers use antiparasitic drugs, $63.5 \%$ use drugs and natural antiparasitic products and $6.1 \%$ use natural antiparasitic products. The natural products used are: paico and "leche de ojé" in $27 \%$, paico and papaya seed in $17.4 \%$, warm drink of "hierba buena" with milk and use of the paico in $14.8 \%$, pumpkin seed and warm drink of "hierba buena" in $10.4 \%$. The way of acquiring knowledge of mothers about the antiparasitic properties of natural products are: $58.3 \%$ have transmitted knowledge of family and friends and $11.3 \%$ report having read about the subject. The preference between the use of drugs and the use of natural antiparasitic products, $20.9 \%$ of mothers prefer the use of drugs for ease of administration and medical indication. Likewise $58.3 \%$ prefer natural products for the use and recommendation of relatives. In conclusion, most mothers use natural products and drugs for deworming their children.
\end{abstract}

Keywords: Drugs, antiparasitic, medicinal plants.

\footnotetext{
${ }^{1}$ Universidad Nacional Toribio Rodríguez de Mendoza de Amazonas, Perú. Email: carla.ordinola@untrm.edu.pe

${ }^{2}$ Universidad Nacional Toribio Rodríguez de Mendoza de Amazonas, Perú. Email: yshoner.silva@untrm.edu.pe

${ }^{3}$ Universidad Nacional Toribio Rodríguez de Mendoza de Amazonas, Perú. Email: oscar.oc@untrm.edu.pe

${ }^{4}$ Universidad Nacional Toribio Rodríguez de Mendoza de Amazonas, Perú. Email: yudelly.torrejon.@untrm.edu.pe

${ }^{5}$ Universidad Nacional Toribio Rodríguez de Mendoza de Amazonas, Perú. Email: oscar.pizarro@untrm.edu.pe
} 


\section{INTRODUCCIÓN}

La parasitosis es un tema de salud pública, que afecta a las personas a nivel mundial, nacional, regional y local. Esta enfermedad es considerada como una Enfermedad Transmitida por Alimentos (ETA), que tiene diversos factores, entre ellos cabe mencionar el consumo de alimentos contaminados, inadecuada higiene intradomiciliaria y deficientes practicas saludables. (OMS)

Las parasitosis siguen siendo un grave problema de salud en pediatría, no tanto por la mortalidad que ocasionan sino por la morbilidad y sus secuelas en el crecimiento y desarrollo de los niños y en su desempeño en la vida adulta (Ochoa LC, 2019).

El Ministerio de Salud, en el boletín epidemiológico 2019, de la Semana 8, señala que las ETA son identificadas como infecciones e intoxicaciones alimentarias. Las infecciones alimentarias son producidas por la contaminación con agentes microbianos de bacterias, virus, hongos y parásitos; mientras que las intoxicaciones son producidas por la ingesta de toxinas preformadas (por animales, vegetales o microorganismos) o sustancias químicas que se incorporan en los alimentos de modo accidental, incidental o intencional en cualquier fase de la cadena alimentaria. Se estiman que alrededor de 250 son los agentes causantes de las ETA (bacterias, virus, parásitos o sustancias químicas).

Anualmente, una de cada cuatro personas presenta algún episodio de ETA y los factores que incrementan el riesgo de exposición de la población son la urbanización acelerada, la necesidad del consumo de alimentos fuera del domicilio, entre otros. En el Perú fue notificado en los últimos 5 años a través del sistema de vigilancia epidemiológica, un promedio de 45 brotes de ETA. (MINSA, 2019) El Perú, es un país rico en diferentes especies naturales. En su diversidad de flora, destacan las plantas medicinales, utilizadas desde tiempos muy antiguos para combatir las enfermedades que se presentaban. En cuanto a plantas medicinales nos enfocamos en las que poseen propiedades antiparasitarias. Como son el paico, árbol de ojé, hierba buena y las semillas de papaya y de zapallo. Productos naturales conocidos por sus respectivas propiedades como laxante y de desparasitación (Casamayor, Pérez, Morales. 2014).

Los productos naturales en mención tienen formas específicas y particulares de preparación y consumo. El paico (Chenopodium abrosioides), arbusto que puede alcanzar $1 \mathrm{~m}$ de altura, muy ramificada en la base y con pubescencia glandular. Posee hojas alternas de color verde oscuro, siendo las inferiores ovoides y lanceoladas y de bordes dentados, y las superiores pequeñas, lanceoladas y de bordes enteros. Sus flores son pequeñas y se agrupan en panículas y su fruto globoso tiene una semilla color negro. Crece de manera silvestre y cultivada en la costa, sierra y Amazonía del Perú, hasta los 4,000 msnm. (Puma R., 2019). 
El paico se puede utilizar como verdura en sopas, chupes y caldos, las semillas pueden utilizarse como aderezo. En el uso medicinal del paico, las hojas son apropiadas para aliviar los cólicos estomacales, resfríos, espasmos, hemorroides, pulmonías, gastritis, dismenorrea, inflamaciones de las vías urinarias, y sirve como antitusígeno, antihelmíntico, purgante, diurético, hepatoprotector, antinflamatorio, antiemético, antiséptico, digestivo y antirreumático. (Chevallier, A., 1997)

Tiene muchas propiedades medicinales en decocción o infusión de hojas y/o la raíz (digestiva, carminativa, tónica y diurética). El Zumo de sus hojas es utilizado como antihelmínitico gracias a sus componentes ascaridol (1,4-peróxido-p-menteno-2) o alcanfor de quenopodio, silvestreno y safrol. En su forma de uso u consumo, el zumo de las hojas de paico en la forma tradicional en la zona: el estracto obtenido triturando y experimentando las hojas sin adicionar agua, se toma en ayunas por 3 a 9 días seguidos y se puede acompañar por algún líquido dulce (miel, agua azucarada) que disimule el sabor característico. (Giove R., 1996)

El árbol de ojé (Ficus insípida), es un árbol de aproximadamente $18 \mathrm{~m}$ de altura a más, tronco recto, copa amplia y frondosa, corteza firme y lisa de color gris parduzco con fisuras paralelas y abundante látex de color blanco-lechoso. Sus hojas son enteras y tienen estípulas terminales. Posee flores bisexuales y un fruto globoso de unos 2 a $3 \mathrm{~cm}$ de diámetro con semillas pequeñas y abundantes. (Giove R., 1996)

El látex blanquecino que produce la corteza del ojé posee múltiples propiedades y se lo utiliza como purgante, antihelmíntico, antirreumático, hematopoyético, depurativo de la sangre, para el dolor de muelas, picadura de hormiga, mordedura de serpiente y contra la temible uta. Las hojas de esta especie se utilizan como antiemético, antipirético, febrífugo y antianémico. Los frutos son un buen mnemónico, es decir, estimulan la memoria. (Chevallier, A., 1997)

La hierba buena (Menta piperita), también conocida como menta verde o hierba santa, se puede combinar con leche para obtener un remedio antiguo, efectivo y natural para eliminar los parásitos intestinales, siguiendo pautas particulares de alimentación. Esta combinación se consume tibia, en ayunas, esperado media hora después de su consumo para ingerir alimentos sólidos libre de condimentos, azúcares, bebidas alcohólicas y de lácteos y sus derivados. (Agüero ME., De la Cruz F., Prieto A. \& Miniet A., 2012)

Las semillas de papaya, son de forma redonda, aspecto brillante y sabor ligeramente picante. Resultan ser un excelente remedio para eliminar parásitos intestinales. Cuya preparación consiste en batir aproximadamente 40 semillas junto con la pulpa de la papaya. Batido que debe ser consumido media hora antes del desayuno. Otra forma de preparación es dejando secar las semillas y pulverizarlas, para luego mezclarlas con miel de abeja. Esta preparación se consume tres veces al día con una dosis de 
una cucharada sopera, media hora antes de cada comida, junto con un vaso de agua y el jugo de medio limón.(Agüero ME., De la Cruz F., Prieto A. \& Miniet A., 2012)

Las semillas de calabaza contienen vitaminas A, B, E, C, K, cal y fósforo; en las semillas hay en abundante proporción un aceite compuesto por glicéridos de ácidos linólico, palmítico, esteárico y oleico; contiene también zinc y fósforo, globulina, sacarosa, lecitina, albuminoides, fermentos, peporresina, resinas y azúcar. Entre sus propiedades se encuentran las antiescorbúticas, refrescantes, suavizantes, pectorales, tónicas cardíacas, remineralizantes, laxantes, antidiarreicos, antihelmínticas, tónico hormonales, emolientes, calmantes, diuréticas, febrífugas, tenífugas, afrodisíacas. (Agüero ME., De la Cruz F., Prieto A. \& Miniet A., 2012)

Como aplicaciones terapéuticas se emplea en el tratamiento de la tenía, parásitos intestinales, botriocéfala, ascáride, inflamación de las vías urinarias, contusiones, excoriaciones, quemaduras, hipertrofia de la próstata, diabetes, estreñimiento, diarreas, gases intestinales, dispepsias, inflamaciones del hígado, disentería, enteritis, fiebres, hidropesía, hemorroides, mareos, vómitos, insomnios, afecciones cardíacas. (Agüero ME., De la Cruz F., Prieto A. \& Miniet A., 2012)

A nivel local, en la ciudad de Chachapoyas, existe los productos naturales descritos líneas arriba, sin embargo, es evidente que aún el consumo de estos productos naturales es deficiente, siendo importante la revaloración de dichos productos dentro de la localidad, por sus importantes beneficios para la salud, en particular, como antiparasitarios que otorgan la posibilidad de mejorar el estado nutricional de las personas, en especial de los niños, como grupo vulnerable a padecer de parasitosis. Resulta importante recalcar que los productos naturales con propiedades antiparasitarias son una excelente opción para combatir enfermedades como la parasitosis que genera desnutrición y malestar en niños y en quienes lo padecen.

Considerando esta realidad problemática nos impulsa a investigar sobre el uso de fármacos en relación a productos naturales con propiedades antiparasitarias en la localidad de Chachapoyas, considerando que es un lugar donde se expresa cultura y se conservan las costumbres de antaño.

\section{MATERIALES Y MÉTODOS}

La muestra estuvo conformada por 115 madres de niños en etapa escolar del distrito de Chachapoyas.

\section{Criterios de inclusión:}

Madres de niños y niñas en etapa escolar del distrito de Chachapoyas, que participen de manera voluntaria.

\section{Criterios de Exclusión:}

Madres de niños y niñas en etapa escolar del distrito de Chachapoyas que no tuvieron la voluntad de participar en el presente trabajo de investigación. 


\section{Instrumento}

Para obtener la información básica a fin de cumplir con los objetivos propuestos, se utilizó como método la entrevista y como instrumento el cuestionario. La aplicación del instrumento se realizó en los hogares de las madres y nos permitió analizar y cuantificar el uso de fármacos y productos naturales antiparasitarios. La aplicación del instrumento fue de carácter individual y se aplicó en aproximadamente 20 minutos, en las viviendas de las madres participantes.

\section{Métodos y Técnica}

Método: El método que se utilizó fue la encuesta.

Técnica e instrumentos de recolección de datos. Para medir la variable en estudio se utilizó la técnica del cuestionario, que ayudó a la recopilación de datos a través de preguntas abiertas y cerradas (Hernández, 2010).

\section{RESULTADOS}

En la Figura 1 a la Figura 4 se muestran el uso de fármacos y de productos naturales, las formas de adquisición del conocimiento y los motivos de preferencia entre el uso de fármacos o el uso de productos naturales antiparasitarios.

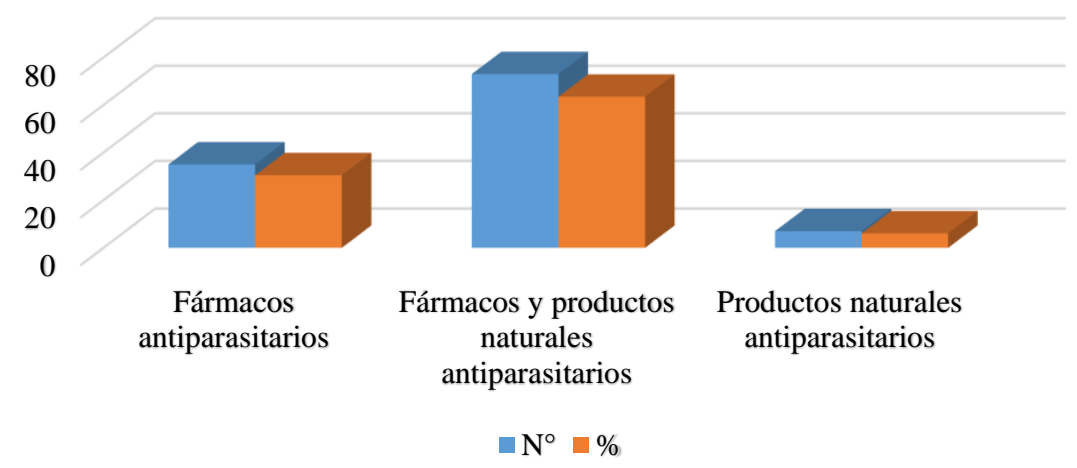

Figura 1. Uso de fármacos y de productos naturales antiparasitarios en niños en etapa escolar

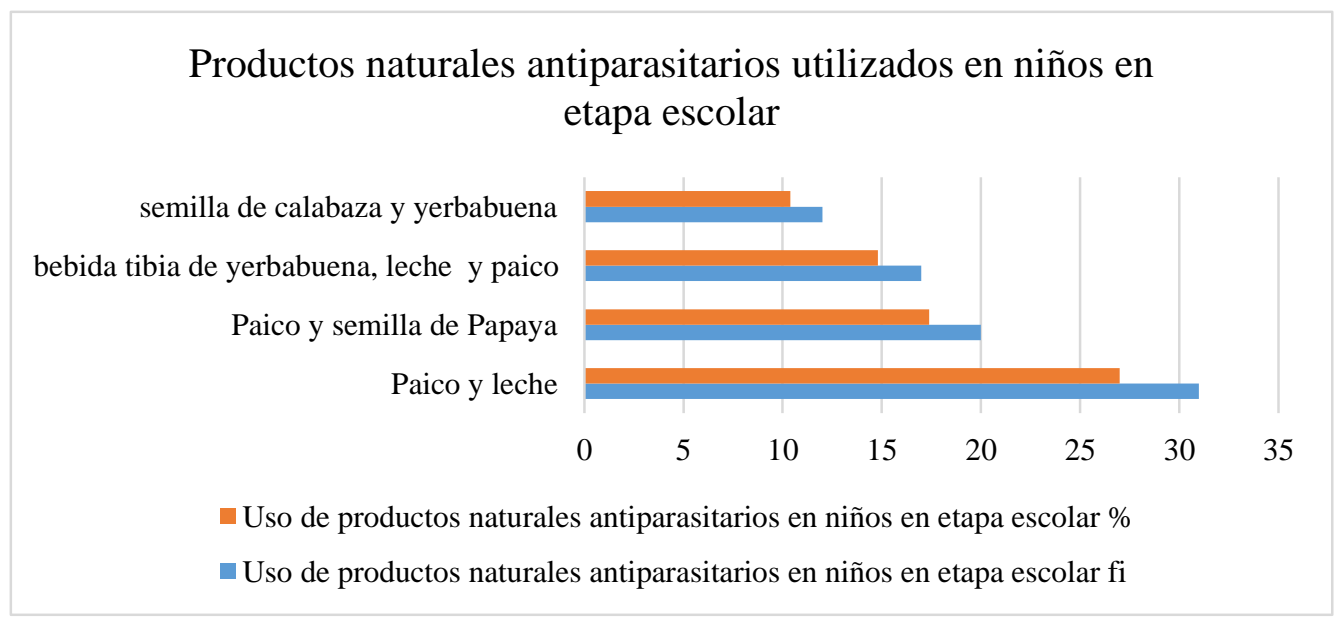

Figura. 2. Productos naturales antiparasitarios utilizados en niños en etapa escolar. Chachapoyas, 2019. 


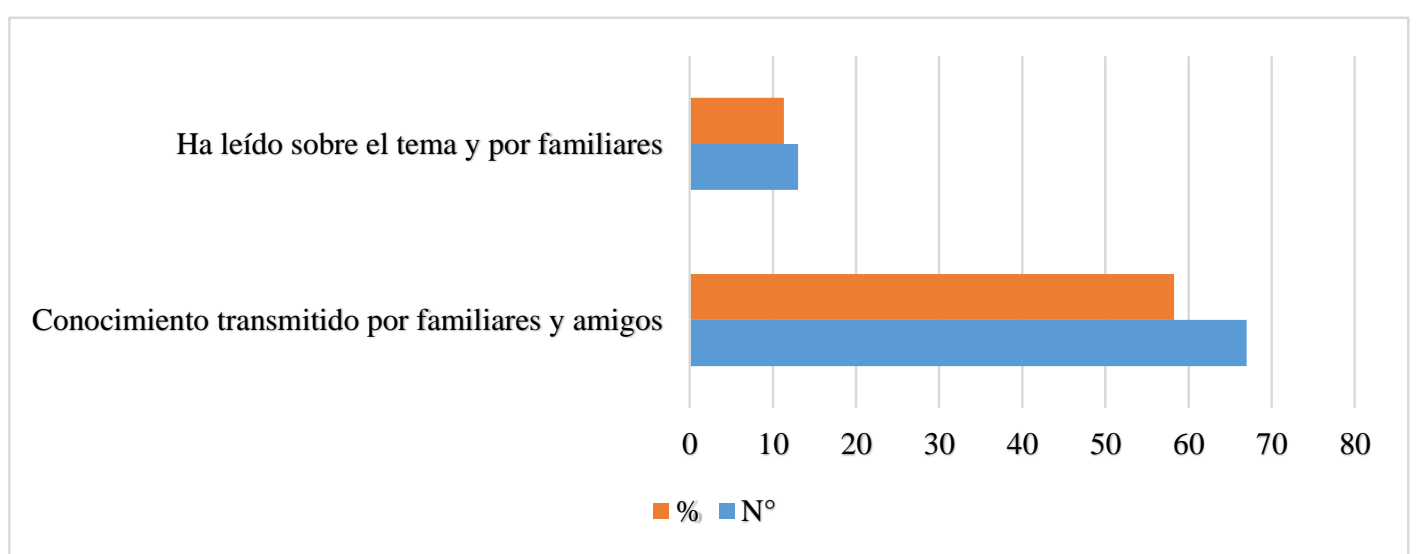

Figura 3. Formas de adquisición de conocimiento sobre las propiedades antiparasitarias de los productos naturales.

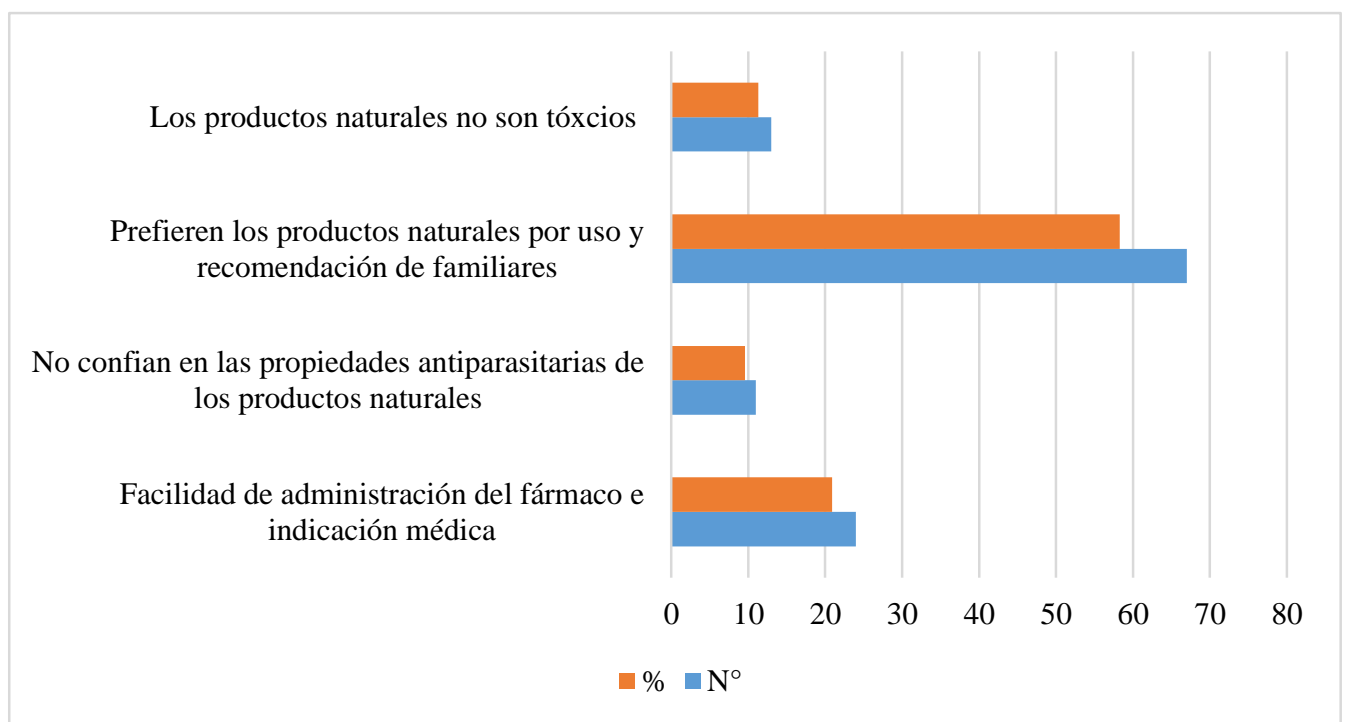

Figura 4. Motivos de preferencia entre el uso de fármacos y el uso de productos naturales antiparasitarios.

\section{DISCUSIÓN}

En la Figura 1, sobre el porcentaje entre el uso de fármacos y el uso de productos naturales antiparasitarios en niños en etapa escolar, muestra que el 30.4\% (35), utiliza solo fármacos antiparasitarios, una clara muestra que los productos farmacéuticos están cobrando mayor relevancia sobre los productos naturales antiparasitarios. Una importante mayoría el 63.5\% (73) utiliza tanto fármacos como productos naturales antiparasitarios, este resultado refleja que las madres de familia no confían en los productos naturales antiparasitarios, además este resultado muestra que la utilización de productos naturales ancestrales antiparasitarios heredados de generación en generación va perdiendo utilidad dando paso a los productos quimico-farmaceuticos y en un porcentaje minoritario el $6.1 \%$ (7) utiliza solo productos naturales antiparasitarios, este pequeño porcentaje de madres de familia aún conserva los conocimientos ancestrales de plantas medicinales usadas en la desparasitación de sus menores hijos. 
Con respecto a lo descrito, Samaniego N. \&Tenesaca JJ. (2019), en su trabajo de investigación titulado Paico y Albendazol en el manejo de ascaridiasis y giardiasis en escolares de la escuela Rumiñahui de San Lucas, Loja -Ecuador; señala que el tratamiento con paico y albendazol resultó ser altamente eficaz en el manejo terapéutico de ascaridiasis, sin embargo, para el tratamiento de giardiasis ambos fueron efectivos parcialmente. Tanto el Paico como Albendazol se pueden administrar de forma segura, ya que no se evidenciaron efectos adversos con ninguno de los tratamientos administrados.

En la Figura 2, donde se muestra los productos naturales antiparasitarios utilizados en niños en etapa escolar. El paico y la "leche de ojé" son productos naturales de mayor uso en un 27\% (31), el paico y la semilla de papaya en $17.4 \%$ (20), la bebida tibia de hierba buena con leche y uso del paico en $14.8 \%$ (17), la semilla de calabaza y bebida tibia de hierba buena en $10.4 \%$ (12). Respecto al resultado presentado, Quispe M. \& Quispe C. (2018), establecen que los pobladores del Barrio del distrito de Pucará - Huancayo, utilizan el ajenjo, el paico y el zapallo ante dolencias causadas por parasitosis. Estas plantas medicinales son utilizadas según el tipo de parasitosis y representan una alternativa de tratamiento.

Los resultados de la figura anterior se relacionan con lo presentado en la Figura 3, donde se expone la forma de adquisición de conocimientos de las madres participantes sobre las propiedades antiparasitarias de los productos naturales, donde se muestra que el 58.3\% (67) poseen conocimientos transmitido de familiares y amigos y el 11.3\% (13) refiere haber leído sobre el tema y por familiares. Respecto a lo detallado, Mejía K. \& Rengifo E. (2000) citado por Quispe M. \& Quipe C. (2018), señalan que el conocimiento de las propiedades medicinales de las plantas está basado en la observación, la experiencia y el conocimiento profundo del entorno, los cuales serán transmitidos de generación en generación y serán enriquecidos por la integración cultural de la población nativa y migrante, este saber se ha obtenido de la medicina popular y la herboristería actual. Estos conocimientos que se encuentran debidamente sistematizados, deben contribuir a resolver, en parte, los problemas de salud de la población menos favorecida y más alejada de la modernidad, cuyas posibilidades de curarse son, actualmente, limitadas por el alto costo de los fármacos modernos.

En la Figura 4, se presentan los motivos de preferencia de las madres de niños y niñas en etapa escolar entre el uso de fármacos y el uso de productos naturales antiparasitarios, destacando que el $20.9 \%$ (24) de madres participantes manifiestan que prefieren darle el fármaco por la facilidad de administración e indicación médica. El 9.5\% (11) indican que no confían en la eficacia de las propiedades antiparasitarias de los productos naturales. Sin embargo, el 58.3\% (67) manifestaron que prefieren los productos naturales por uso y recomendación de familiares. El 11.3\% (13) indican que 
principalmente prefieren los productos naturales por no ser tóxicos para sus niños y niñas (no causan daño).

Con respecto a lo descrito, Álvarez \& col. (2015) citado por Torres RC. \& Vivar ET. (2016), en su investigación sobre el Estandarización de un bioensayo de actividad antiparasitaria utilizando como organismo modelo a Fasciola hepática. El uso de plantas con actividad antihelmíntica puede ser una alternativa al control de los parásitos. La búsqueda de compuestos bioactivos con propiedades antihelmínticas aumenta significativamente debido a que, metabolitos secundarios son los compuestos más importantes como nuevas alternativas para el control de parásitos.

Por su parte, Ochoa LC. (2019), en su estudio sobre parasitosis y antiparasitarios en niños, menciona que actualmente se dispone de antiparasitarios efectivos, fáciles de suministrar (incluso por personal no médico). Los más comunes (Albendazol y Mebendazol) actúan inhibiendo la síntesis de adenosín trifosfato (ATP) necesario para su supervivencia. Como solamente se absorbe entre el 1\% y el 5\% del medicamento y su metabolismo es rápido, los efectos secundarios son leves y transitorios.

Según los resultados presentados, cabe recalcar que los productos naturales necesitan ser revalorados y difundidos como valiosas alternativas de tratamiento para los casos de parasitosis, cuyo costo es mucho menor que el de los fármacos. La eficacia antiparasitaria del uso de los productos naturales descritos ha sido comprobada por las personas que lo han utilizado y lo siguen utilizando.

\section{CONCLUSIONES}

Una importante mayoría de madres refieren brindar tanto fármacos como productos naturales para combatir la parasitosis en los niños y niñas en etapa escolar. El Paico y la Leche de Ojé, son los productos naturales de mayor consumo en los niños en etapa escolar. El conocimiento sobre las propiedades antiparasitarias de los productos naturales, en más de la mitad de las madres participantes, lo han adquirido de familiares y amigos.

Una mayoría significativa de madres de niños y niñas en etapa escolar prefieren los productos naturales antiparasitarios por uso y recomendación de sus familiares. Además, señalan que son productos que no causan daño a sus niños y niñas.

La totalidad de madres que ha utilizado y utiliza los productos naturales antiparasitarios manifiestan la eficacia de los mismos. 


\section{REFERENCIAS BIBLIOGRÁFICAS}

Agüero M, De la Cruz F, Prieto A. \& Miniet A. (2012). La Semilla de Calabaza como Terapéutica Antiparasitaria en la Población de 5 a 9 años. Zamuro. Tucupita. Revista Multimed. No. 4 $16(4)$

Torres R. \& Vivar E. (2016) Estandarización de un Bioensayo de Actividad Antiparasitaria Utilizando como Organismo Modelo a Fasciola Hepática. (Tesis de titulación). Universidad De Cuenca Facultad de Ciencias Químicas, Carrera de Bioquímica y Farmacia. Cuenca Ecuador. Recuperado de: http://dspace.ucuenca.edu.ec/handle/123456789/25521

Ministerio de Salud (2019). Boletín Epidemiológico. Semana 8. ISSN2415-076 2

Disponible en: https://www.dge.gob.pe/portal/docs/vigilancia/boletines/2019/

Giove R, 1996. Medicina Tradicional en el Tratamiento de Enteroparasitosis. Revista de Gastroenterología del Perú - Volumen 16, №3 1996

Hernández S, Fernández C., Baptista MP. (2010). Metodología de la Investigación. 5ta Edición. Editorial Mc Graw Hill. ISBN: 978-607-15-0291-9 (ISBN edición anterior: 978-970-105753-7) Recuperado de: https://www.esup.edu.pe/descargas/dep_investigacion/Metodologia\%20de\%20la\%20investi gaci\%C3\%B3n\%205ta\%20Edici\%C3\%B3n.pdf

Ochoa C. (2019). Parasitosis y Antiparasitarios en Niños. Revista Medicina U.P.B., vol 38, num 1. Pp. 46-56. Universidad Pontificia Boliviana Medellin, Colombia. Recuperado de: http://www.redalyc.org/articulo.oa?id=159058103006

Chevallier A. (1997). Enciclopedia de Plantas Medicinales. En Enciclopedia de Plantas Medicinales (pág. 186). España: Acento Editorial- Madrid.

Puma R. (2019). Extracción y Caracterización de Aceite Esencial de Paico (Chenopodium Ambrosioides) Mediante Arrastre de Vapor. (Tesis de titulación). Universidad Nacional del Altiplano. Puno- Perú. Recuperado de: repositorio.unap.edu.pe/bitstream/handle/UNAP/11304/Puma_Mamani_Rider_Yampolh.pdf ?sequence $=1 \&$ is Allowed $=\mathrm{y}$

Quispe M. \& Quispe C. (2018). Plantas Medicinales Utilizadas Como Alternativa de Tratamiento en Parasitosis en los Pobladores Del Barrio del Distrito de Pucará - Huancayo. (Tesis de titulación). Universidad Privada de Huancayo "Franklin Roosevelt". Huancayo - Perú. Recuperado de: http://repositorio.uroosevelt.edu.pe/xmlui/handle/ROOSEVELT/140

Samaniego N. \& Tenesaca JJ. (2019). Paico y Albendazol en el Manejo de Ascaridiasis y Giardiasis en Escolares de la Escuela Rumiñahui de San Lucas, Loja -Ecuador. (Tesis de titulación). Universidad Nacional de Loja. Loja - Ecuador. 